\title{
Diagnóstico de enfermagem mobilidade física prejudicada em pacientes com acidente
}

\section{vascular encefálico}

\author{
Nursing diagnosis impaired physical mobility in patients with stroke \\ Diagnóstico de enfermería movilidad física alterada en pacientes con ictus
}

Recebido: 25/02/2021 | Revisado: 03/03/2021 | Aceito: 07/03/2021 | Publicado: 15/03/2021

\author{
Andreza Cason \\ ORCID: https://orcid.org/0000-0001-5598-6223 \\ Hospital Regional do Oeste, Brasil \\ E-mail: andreza_cason@hotmail.com \\ Olvani Martins da Silva \\ ORCID: https://orcid.org/0000-0002-4285-3883 \\ Universidade do Estado de Santa Catarina, Brasil \\ E-mail: olvani.silva @udesc.br \\ Gloriana Frizon \\ ORCID: https://orcid.org/0000-0002-6760-9465 \\ Hospital Regional do Oeste, Brasil \\ E-mail: gloria.ana.cco@gmail.com \\ Jussara de Lima \\ ORCID: https://orcid.org/0000-0002-2375-9584 \\ Hospital Regional do Oeste, Brasil \\ E-mail: jhudlima@hotmail.com \\ Carolyne Diehl Stuani \\ ORCID: https://orcid.org/0000-0002-7864-4776 \\ Hospital Regional do Oeste, Brasil \\ E-mail: carolynedi@gmail.com
}

\begin{abstract}
Resumo
Objetivos: investigar a ocorrência do diagnóstico de enfermagem mobilidade física prejudicada, suas características definidoras e fatores relacionados, em pacientes com acidente vascular encefálico em um hospital público. Método: estudo documental em prontuários de 40 pacientes internados no setor de neurologia com diagnóstico de acidente vascular encefálico, a coleta de dados ocorreu entre os meses de maio à julho de 2020, por meio de um instrumento que constam dados sociodemográficos e relacionados ao processo de enfermagem. Utilizou-se análise de dados descritiva. Resultados: houve predomínio do sexo masculino, idosos, hipertensos, 50\% dos prontuários constava o processo de enfermagem, destes apenas $17,5 \%$ apresentavam o diagnóstico de enfermagem mobilidade física prejudicada elencado. As principais características definidoras citadas foram: redução nas habilidades motoras finas e grossas e dificuldade para virar-se, como fatores relacionados, a força muscular diminuída e alteração na função cognitiva foram os mais elencados. Conclusão: o diagnóstico de mobilidade física prejudicada foi pouco expressivo e a característica definidora esteve focada na redução das habilidades motoras, relacionado a força muscular diminuída. Palavras-chave: Diagnóstico de enfermagem; Acidente vascular encefálico; Limitação da mobilidade.
\end{abstract}

\begin{abstract}
Objectives: to investigate the occurrence of the nursing diagnosis of physical mobility impaired, its defining characteristics and related factors in patients with stroke in a public hospital. Method: documentary study on medical records of 40 patients admitted to the neurology sector with a diagnosis of stroke, between May and July 2020, with collection of sociodemographic data and related to the nursing process. Descriptive data analysis was used. Results: there was a predominance of males, the elderly, hypertensive patients, $50 \%$ of the medical records contained the nursing process, of which $17.5 \%$ had a nursing diagnosis of impaired physical mobility. The main related defining characteristics were: reduction in fine and gross motor skills and difficulty in turning around, as related factors, decreased muscle strength and altered cognitive function. Conclusion: the diagnosis of impaired physical mobility was not very significant and the defining characteristic was focused on the reduction of motor skills, related to decreased muscle strength.
\end{abstract}

Keywords: Nursing diagnosis; Brain stroke; Mobility limitation.

\section{Resumen}

Objetivos: investigar la ocurrencia del diagnóstico de enfermería de movilidad física deteriorada, sus características definitorias y factores relacionados, en pacientes con ictus en un hospital público. Método: estudio documental sobre 
historias clínicas de 40 pacientes ingresados al sector de neurología con diagnóstico de ictus, la recolección de datos se realizó entre los meses de mayo a julio de 2020, utilizando un instrumento que contiene datos sociodemográficos y relacionados con el proceso de enfermería. Se utilizó análisis de datos descriptivos. Resultados: hubo predominio del sexo masculino, ancianos, hipertensos, el 50\% de las historias clínicas contenía el proceso de enfermería, de las cuales solo el 17,5\% tenía el diagnóstico de enfermería de movilidad física alterada. Las principales características definitorias citadas fueron: reducción de la motricidad fina y gruesa y dificultad para dar la vuelta, ya que los factores relacionados, la disminución de la fuerza muscular y los cambios en la función cognitiva fueron los más enumerados. Conclusión: el diagnóstico de alteración de la movilidad física fue poco significativo y la característica definitoria se centró en la reducción de la motricidad, relacionada con la disminución de la fuerza muscular.

Palabras clave: Diagnóstico de enfermería; Accidente cerebrovascular; Limitación de la movilidad.

\section{Introdução}

O AVE pode ser ocasionado por um trombo ou êmbolo quando na forma isquêmica ou pelo rompimento de um vaso sanguíneo no encéfalo caracterizado como hemorrágico. Ambos causam disfunção cerebral, contudo, a forma como ocorre a lesão é diferenciada (Lima, et al., 2016). Dentre os principais sinais e sintomas cita-se o formigamento ou fraqueza na face ou no braço, especialmente em um lado do corpo, confusão mental, alteração da fala, desvio da rima labial, dor de cabeça súbita, intensa e sem causa aparente (Ministério da Saúde, 2020).

Dentre as diversas sequelas decorrentes do AVC, há presença da hemiparesia ou hemiplegia, que afeta o equilíbrio e consequentemente a mobilidade física da pessoa, assim como a força muscular insuficiente, acrescida de prejuízo neuromuscular, que prejudica ou até mesmo impossibilita tarefas simples do dia a dia, como manter-se sentado, passar de sentado para de pé e deambular (Lima, 2020).

As complicações decorrentes do acidente vascular encefálico em relação ao equilíbrio e movimento variam de acordo com a localização da lesão, do tempo de perfusão inadequada e da existência de circulação colateral. Diante das manifestações clínicas oriundas da doença, a equipe de saúde, especialmente o enfermeiro, tem papel fundamental no planejamento e implementação de cuidados que englobe as necessidades manifestadas pelo cliente e contribua dessa forma para sua reabilitação, sendo possível para isto, a utilização do processo de enfermagem (Nunes, Fontes, \& Lima, 2016).

$\mathrm{O}$ processo de enfermagem (PE) tem-se caracterizado como a principal ferramenta metodológica para o desenvolvimento sistematizado da prática profissional, ao possibilitar um julgamento clínico por meio do diagnóstico de enfermagem (DE), avaliação e intervenções propostas (Herdtman, \& Kamitsuru, 2018).

Dentre os possíveis diagnósticos encontra-se o de mobilidade física prejudicada (MFP), descrito como uma dificuldade de movimentação voluntária e independente, de um ou mais membros do corpo, sendo a limitação da mobilidade uma das sequelas mais presentes no paciente pós AVE (Herdtman, \& Kamitsuru, 2018).

Nesse sentido é fundamental identificar as habilidades prejudicadas e os déficit adquiridos pelo paciente pós AVE, para propor uma reabilitação precoce da mobilidade equilíbrio, marcha e força que, além de melhorarem a mobilidade do indivíduo, ainda diminuem a ocorrência de quedas e favorece a autonomia (Cavalcante, Nemer, Moreira, \& Ferreira, 2018).

Embora seja valioso o desenvolvimento e aplicação do processo de enfermagem, destaca-se a escassez de pesquisas desenvolvidas no Brasil referente a diagnósticos de enfermagem em pacientes que sobreviveram ao acidente vascular encefálico. Estudar diagnósticos de enfermagem, em particular a mobilidade física prejudicada em sobreviventes desse agravo de saúde, possibilita planejar e implementar estratégias de segurança ao paciente, afim de evitar eventos adversos, promover a inserção precoce do indivíduo a sociedade e contribuir com as evidências científicas (Lima et al., 2016).

Mediante a esse propósito a questão norteadora que impulsionou o presente estudo foi delimitada da seguinte forma: qual a ocorrência do diagnóstico de enfermagem mobilidade física prejudicada, em pacientes com acidente vascular encefálico internados no setor de neurologia de um hospital público? E trouxe como objetivos específicos, identificar as principais características definidoras e fatores relacionados ao diagnóstico de enfermagem mobilidade física prejudicada. 


\section{Metodologia}

Estudo quantitativo exploratório, com coleta de dados nos meses de maio à julho de 2020, no setor de neurologia de um hospital público no oeste de Santa Catarina.

A escolha pela pesquisa quantitativa se relaciona com as atribuições ao final da análise dos dados, pois é por meio de teorias objetivas que são examinadas as variáveis e suas relações, possibilitando as análises através de instrumentos (Creswell, 2010). Por meio da metodologia quantitativa, após a coleta de dados obtém-se dados que permitem serem visualizados na forma de estatísticas, porcentagens, com uso de tabelas e gráficos para melhor compreensão dos resultados (Pereira, Shitsuka, Parreira, \& Shitsuka 2018).

Ela é um tipo de pesquisa que utiliza diferentes técnicas para mensurar informações referentes a um determinado estudo/problema, é pautada por variáveis quantificadas em números, sendo analisadas através de estatísticas, com intuito de determinar se os conceitos descritos na teoria se sustentam ou não (Knechtel, 2014).

A pesquisa exploratória é uma forma de pesquisa utilizada quando o tema em estudo é pouco explorado (Oliveira, 2018). Segundo Gil (2019), as pesquisas exploratórias tem como objetivo possibilitar maior familiaridade com o problema, com intuito de torná-lo mais evidente ou a construir hipóteses.

O setor em estudo possui 32 leitos, com uma taxa de ocupação média de 104 pacientes ao mês, independente do Código Internacional de Doenças (CID). Atuam na unidade quatro enfermeiras assistenciais, uma enfermeira coordenadora e 19 técnicos de enfermagem.

O sistema de dados para organizar e gerenciar as informações dos prontuários de forma eletrônica é G-Hosp, que está em processo de expansão para informatizar todo processo de atendimento do paciente.

Participaram do estudo, pacientes (por meio de seus prontuários) internados no setor de neurologia durante o período de coleta de dados. A amostragem foi por conveniência (Lunardi, 2020). Assim, foram incluídos prontuários de pacientes com diagnóstico médico confirmado de acidente vascular encefálico, maiores de 18 anos, ambos os sexos, com tempo de internação mínima de 24h. Excluiu-se aqueles com diagnóstico médico de AVE que encontravam-se inconscientes, totalizando um n=40 pacientes.

Foi utilizado um instrumento de coleta contendo variáveis relacionados a caracterização do paciente: sexo, idade, o tipo de AVE, doenças prévias, fatores de risco e na segunda parte dados relacionados ao DE: presença ou não do diagnóstico de enfermagem mobilidade física prejudicada no processo de enfermagem, características definidoras e fatores relacionados que foram elencados.

A coleta ocorreu nos prontuários eletrônico e físico, onde a pesquisadora os analisava em busca do processo de enfermagem e do diagnóstico mobilidade física prejudicadas, duas vezes por semana. A primeira busca ocorria na data da internação e a segunda busca era considerada na data da alta ou óbito.

Os dados foram armazenados no programa Excel®, posteriormente importados para o programa Statistical Package for Social Science (SPSS) versão 22. As variáveis categóricas foram expressas por números e percentuais, as contínuas quando distribuição normal foram apresentadas por média e desvio padrão, ou mediana e intervalo interquartil. O estudo foi aprovado pelo Comitê de Ética em Pesquisa da Universidade Comunitária da Região da Chapecó (UNOCHAPECÓ) sob parecer consubstanciado $\mathrm{n}^{\circ} 4.001 .596 \mathrm{em} 30$ de abril de 2020 .

\section{Resultados}

Dos pacientes do estudo houve prevalência do sexo masculino, com média de idade acima de 60 anos. Com maior índice de AVE isquêmico (AVEi), apresentando como doença crônica prévia a Hipertensão Arterial Sistêmica (HAS) em sua maioria. Em relação a outros fatores de risco que podem propiciar o desenvolvimento de um AVE a maioria não apresentava 
registrado. O tempo e internação dos pacientes no setor de neurologia obteve uma mediana de cinco dias de internação. Dados são apresentados na Tabela 1:

Tabela 1 - Características dos pacientes do estudo. Chapecó- SC, 2020.

\begin{tabular}{ll}
\hline Gênero & $\mathrm{n}=40$ \\
Masculino & $27(68 \%)$ \\
Feminino & $13(32 \%)$ \\
\hline Idade & $64 \pm 14,3$ \\
\hline Tipo de AVE & $24(60 \%)$ \\
Isquêmico & $9(22 \%)$ \\
Hemorrágico & $7(17 \%)$ \\
Não especificado & \\
Doença Crônica Prévia & $15(38 \%)$ \\
\hline Hipertensão Arterial & $13(32 \%)$ \\
Hipertensão e Diabetes & $12(30 \%)$ \\
Não especificado & \\
\hline Outros fatores de risco & $1(2 \%)$ \\
Alcoolismo & $3(8 \%)$ \\
Tabagismo & $3(8 \%)$ \\
Alcoolismo e Tabagismo & $1(2 \%)$ \\
Triglicerídeos & $32(80 \%)$ \\
Sem fator de risco especificado & $5(4-10)$ \\
\hline Dias de internação &
\end{tabular}

Nota: Variáveis categóricas expressas como n (\%) contínuas \pm DP e (P25-P75).

Fonte: Autores (2020).

Analisando os pacientes em relação ao gênero para determinar a ocorrência de AVE e os fatores de risco, observa-se que o sexo feminino apresentou maior índice de ocorrência do AVE do tipo isquêmico enquanto que o sexo masculino apresentou maior índice de do AVE do tipo hemorrágico.

Quanto ao diagnóstico de doença crônica o sexo masculino obteve maior número de casos em relação a hipertensão exclusivamente, entretanto a presença de diabetes associada à hipertensão o sexo feminino apresentou maior número. $\mathrm{O}$ sexo masculino foi o único a apresentar fatores de risco elencados, sendo o tabagismo exclusivamente seguido do tabagismo associado ao alcoolismo. Como mostra a Tabela 2:

Tabela 2 - Relação do Gênero com o tipo de AVE, DCNT e fatores de risco. Chapecó- SC, 2020.

\begin{tabular}{ll|l|l}
\cline { 3 - 4 } & & \multicolumn{2}{c}{ Gênero } \\
\hline V|ariáveis & & Feminino & Masculino \\
\hline \multirow{2}{*}{ AVE } & Isquêmico & $10(25 \%)$ & $14(35 \%)$ \\
\cline { 2 - 4 } & Hemorrágico & $3(7 \%)$ & $6(15 \%)$ \\
\cline { 2 - 4 } & Não especificado & 0 & $7(18 \%)$ \\
\hline \multirow{2}{*}{ FCNT prévia } & Hipertensão & $3(7 \%)$ & $12(30 \%)$ \\
\cline { 2 - 4 } & Hipertensão + Diabetes & $6(15 \%)$ & $7(18 \%)$ \\
\cline { 2 - 4 } & Não especificado & $4(10 \%)$ & $8(20 \%)$ \\
& Alcoolismo & 0 & $1(2 \%)$ \\
\cline { 2 - 4 } & Tabagismo & 0 & $3(7 \%)$ \\
\cline { 2 - 4 } & Triglicerídeos & 0 & $1(2 \%)$ \\
\cline { 2 - 4 } & Alcoolismo e Tabagismo & 0 & $3(7 \%)$ \\
\cline { 2 - 4 } & Sem fator de risco & $13(33 \%)$ & $19(48 \%)$ \\
\hline
\end{tabular}

Nota: Variáveis categóricas expressas como n (\%).

Fonte: Autores (2020). 
Ao explorar a presença do PE no prontuário dos pacientes do estudo, observou-se que em $50 \%$ não constava o PE desenvolvido, daqueles que possuíam o PE 17,5\%, constava o diagnóstico de enfermagem MFP elencado.

Dentro do diagnóstico de enfermagem MFP, encontrou-se como características definidoras mais citadas: redução nas habilidades motoras finas e grossas, dificuldade para virar-se, alteração na marcha e movimentos descoordenados. Com menor proporção foi observado a presença de movimentos lentos, desconforto e instabilidade postural. Conforme apresentado na Tabela 3:

Tabela 3 - Características definidoras do diagnóstico mobilidade física prejudicada encontradas no estudo. Chapecó- SC, 2020.

\begin{tabular}{lc}
\hline Característica Definidora & $\mathbf{n}(\%)$ \\
\hline Alteração na marcha & $4(14,8 \%)$ \\
\hline Dificuldade para virar-se & $5(18,5 \%)$ \\
\hline Instabilidade postural & $2(7,4 \%)$ \\
\hline Movimentos descoordenados & $4(14,8 \%)$ \\
\hline Redução nas habilidades motoras finas & $6(22,2 \%)$ \\
\hline Redução nas habilidades motoras grossas & $6(22,2 \%)$ \\
\hline Movimentos lentos & $3(11,1 \%)$ \\
\hline Desconforto & $3(11,1 \%)$ \\
\hline
\end{tabular}

Nota: Variáveis categóricas expressas como $\mathrm{n}(\%)$.

Fonte: Autores (2020).

Assim como possui características definidoras dentro do diagnóstico de enfermagem, também encontrou-se os fatores relacionados. No presente estudo foi verificado a ocorrência do fator relacionado força muscular diminuída e alteração na função cognitiva como os mais presentes dentro do diagnóstico de MPF. Como é possível observar na Tabela 4:

Tabela 4 - Fator relacionado ao diagnóstico mobilidade física prejudicada presente nos diagnósticos de enfermagem dos pacientes do estudo. Chapecó- SC, 2020.

\begin{tabular}{lc}
\hline Fator Relacionado & $\mathbf{n}(\boldsymbol{\%})$ \\
\hline Alteração na função cognitiva & $3(11 \%)$ \\
\hline Alteração na integridade de estruturas ósseas & $1(4 \%)$ \\
\hline Prejuízo neuromuscular & $2(7 \%)$ \\
\hline Dor & $1(4 \%)$ \\
\hline Força muscular diminuída & $4(15 \%)$ \\
\hline Prejuízo musculoesquelético & $1(4) \%)$ \\
\hline
\end{tabular}

Fonte: Autores (2020).

\section{Discussão}

O aumento dos índices de AVE, tem impulsionado interesse por parte de diversos profissionais relacionados à saúde, pela busca por conhecimento técnico e científico que possibilitem práticas clínicas mais efetivas, com resultados eficazes e assim uma qualidade de vida mais adequada a essa população. Com isso, destaca-se a relevância desta pesquisa para a prática de enfermagem, reforçando a falta de estudos sobre os diagnósticos de enfermagem, em especial de mobilidade física prejudicada em sobreviventes de AVE (Nunes, Fontes, \& Lima, 2016).

No presente estudo a maioria dos pacientes eram do sexo masculino, dados semelhantes ao estudo realizado por Moreira et al. (2016) o qual realizou sua pesquisa em um ambulatório de neurologia que é referência para o tratamento de pessoas acometidas por AVE na região nordeste do Brasil. Também vai de encontro com o trabalho desenvolvido por Mourão et al. (2017), que avaliou o perfil de pacientes com AVE em um Hospital de Minas Gerais. 
Em outro estudo desenvolvido por Santos, e Waters (2020) por meio de uma revisão integrativa, também foi evidenciado que o sexo masculino é mais afetado, entretanto, na literatura disponível, observa-se pouca diferença entre os sexos relacionado à incidência do AVE, não sendo classificado como um fator de risco expresso para a doença ser do sexo masculino, tendo em vista que a prevalência do sexo masculino não é expressiva em relação ao sexo feminino.

Referente a faixa etária mais acometida pelo AVE neste estudo, observou-se predominância na população idosa. Individuos idosos acometidos por AVE também foram encontrados no estudo desenvolvido por Barella, Duran, Pires, e Duarte (2019), em um hospital filantrópico no sul de Santa Catarina, onde a idade média dos pacientes foi de 66 anos. O mesmo foi encontrado na pesquisa desenvolvida por Gaspari et al. (2019) com 382 pacientes do qual a média de idade dos pacientes foi de 64 anos.

Na população idosa é mais frequente o acometimento de doenças crônicas, como hipertensão arterial, diabetes, câncer e cardiopatias, a interação entre as alterações característicos do envelhecimento, associadas aos fatores genéticos e ambientais (tabagismo, sedentarimo) favorecem o desenvolvimento do AVE (Santos, \& Waters, 2020).

Em relação a tipologia do AVE, o presente estudo corrobora com os dados encontrados no estudo realizado por Mourão et al. (2017), onde avaliou 223 prontuários de paciente em um Hospital de Minas Gerais sendo que destes, 70\% tiveram AVE do tipo isquêmico e como fator de risco mais presente a hipertensão arterial, no presente estudo $60 \%$ dos pacientes acometidos pelo AVE foi de etiologia isquêmica e a maioria dos pacientes tinham HAS como doença.

No presente estudo ao analisar a presença de doença crônica foi possível observar que referente a HAS, os homens foram os mais acometidos, contudo ao se analisar a presença de DM, as mulheres representam a maioria, embora esse dado seja passível de generalização, patologias que atingem o sistema cerebrovascular ocorre principalmente em pessoas com DM em comparação com pacientes não diabéticos. Sobretudo mulheres diabéticas apresentam maior vulnerabilidade de desencadear um AVE em relação aos homens com DM (Araujo, Souza, Dias, Nepomuceno, \& Cola, 2017).

Em relação ao tempo de internação, foi encontrado uma mediana de cinco dias. Já Mourão et al. (2017), encontrou uma média de 12 dias de internação nos pacientes avaliados. O mesmo ocorreu com Gaspari et al. (2019), onde de um total de 353 pacientes que obtiverm alta com diagnóstico de AVC isquêmico e 29 com AIT em uma unidade de AVE de um hospital terciário, o tempo médio de internação foi de 13,7 dias. Tais dados reforçam que a média de dias de permanência hospitalar dos pacientes foram superiores em relação aos dias de internação no presente campo de estudo, o que reflete em um dado positivo.

Ao analisar a relação gênero e tipo de AVE, observou-se que o sexo feminino foi mais acometido pelo AVEi, enquanto o sexo masculino pelo AVEh, diferentemente dos resultados encontrados por Gonçalves, Feitosa, e Borges (2019), ao descreverem o perfil dos pacientes com AVE em um hospital de referência do Ceará, que encontrou maior incidência do AVEi no sexo masculino, e do AVEh entre o sexo feminino. Ressalta-se que os resultados do presente estudo, não é passível de generalizar os dados devido ao tamanho amostral.

O AVEh é popularmente conhecido como sendo mais grave e de pior prognóstico, contudo ocorre em menor proporção. O rompimento de um vaso sanguíneo resulta em hemorragia e muitas vezes edema cerebral e hipertensão intracraniana, com isso esse sangramento danifica o tecido nervoso, causando lesões irreversíveis nos casos de menor gravidade, já nos casos mais graves pode ocorrer à morte (Musse, 2018).

O AVEh é considerado uma das mais preocupantes emergências médicas em neurologia, ocorre com maior frequência devido a um pico importante da pressão arterial em pacientes que tem HAS que não sabiam e consequentemente não tratavam, ou ainda hipertensos que não se tratam adequadamente (Miranda, 2020).

Com relação a presença de fatores de risco para o desenvolvimento do AVE, nesta pesquisa apenas os homens possuíam, sendo tabagismo unicamente e o tabagismo relacionado ao alcoolismo os mais visualizados. No estudo desenvolvido 
por Albuquerque, Florêncio, Moreira, Lima, Mattos e Ribeiro (2020), que teve como amostra 1073 adultos jovens (20 aos 24 anos) de escolas estaduais de Fortaleza/Brasil, observou-se a presença do fator de risco alcoolismo presente em 36,0\%, e tabagista em 5,5\% dos jovens participantes. Pesquisas apontam que utilizar de maneira exacerbada o álcool e o fumo, propiciam a elevação da pressão arterial, a qual já é elencada como um fator de risco, estimulando assim o desenvolvimento do AVE (Oliveira et al., 2020).

Uma revisão integrativa elaborada por Santos, e Waters (2020), que incluiu oito artigos com o tema relacionado ao perfil epidemiológico do paciente acometido por AVE, mostra que o tabagismo esteve presente em quase 30 por cento dos indivíduos. Costumes como esse elevam em até três vezes a chance de ocorrer um AVE. Referente às doenças cardiovasculares, o tabagismo é extremamente prejudicial aos vasos sanguíneos, provocando danos no endotélio, aumento da formação de coágulos e formação de placas de ateroma. Além disso a nicotina presente no cigarro intensifica a HAS e aumenta a frequência cardíaca.

Outro aspecto a ser considerado, é que os homens costumam dar pouca atenção a sua saúde, bem como à sinais e sintomas pequenos que aparecem no dia a dia. A visão tradicional de masculinidade imposta ao homem como sinônimo de força, bravura em não demonstrar fragilidades, limita a busca pelos serviços de saúde, tendo isso visto como sinal de fraqueza, acham que se preocupar com a saúde não é importante, tornando-se negligentes com sua saúde e bem estar. Isso torna a masculinidade uma das principais barreiras para a busca por serviços de saúde, especialmente para ações de promoção à saúde. Esse é um dos fatores pelo qual as mulheres apresentam maior expectativa de vida em relação aos homens (Teixeira, \& Cruz, 2016; Silva, 2020).

No estudo desenvolvido por Pereira et al. (2015), realizado em uma unidade de saúde da família, no município de João Pessoa, com uma população de estudo composta por homens pertencentes à área de abrangência da referida unidade, apontou que os homens ainda estão pouco presentes nas unidades de saúde, contudo o principal motivo que faz eles irem até este serviço é quando já se encontram com alguma doença instalada, como é o caso da hipertensão.

Referente a elaboração do PE, em metade dos prontuários analisados não havia sido elaborado, durante a coleta de dados observou-se a alta demanda de pacientes internados no setor que tem apenas um enfermeiro assistencial por turno para realizar todas as atividades assistenciais e burocráticas, sendo essa uma das possíveis justificativas pela baixa quantidade de PE desenvolvidos.

No hospital, campo de estudo, o PE ainda está em fase de implantação, ele está disponível no sistema G-Hosp, contudo não são todos os setores do hospital que fazem sua aplicação em 100\% dos pacientes, apenas os setores de UTI (geral, pediátrica e neonatal) que conseguiram se organizar e desempenhar o PE em sua totalidade de pacientes internados. Nos demais setores, incluindo a neurologia, o PE ainda está em fase de implementação, a aplicabilidade varia de acordo com as prioridades e necessidades de cada paciente e setor, bem como da organização, treinamento e orientação de cada coordenador a sua equipe.

A enfermagem tendo como essência o cuidar e a ciência como embasamento científico, deve utilizar referenciais teóricos e metodológicos para nortear a prática clínica. Nessa perspectiva, a Sistematização da Assistência de Enfermagem (SAE) é uma ferramenta de trabalho que promove a qualidade da assistência, com intuito de solucionar os problemas reais e os potenciais riscos de cada indivíduo, sendo um mecanismo importante para a tomada de decisão. Dessa forma, possibilita à equipe de enfermagem elaborar o PE, uma atribuição obrigatória e privativa do enfermeiro (Rosin, Matos, Alves, Carvalho, \& Lahm, 2016).

Tal atividade resulta no estabelecimento dos diagnósticos de enfermagem, por meio do julgamento clínico realizado pelo enfermeiro a fim de assegurar um cuidado assertivo aos pacientes (Herdtman, \& Kamitsuru, 2018). 
Nesse estudo observou-se que uma minoria dos pacientes que tinham PE, tiveram o diagnóstico de MFP elencado, diferentemente dos resultados encontrados no estudo desenvolvido por Lima et al. (2016), o qual realizou um revisão integrativa da literatura em busca de DE vinculados ao paciente acometido pelo AVE, que resultou nos diagnósticos: risco de queda e mobilidade física prejudica, como sendo os mais prevalentes em pacientes no pós AVE. A ocorrência destes diagnósticos explica-se pela doença atingir mais os neurônios motores superiores, que podem afetar o controle dos movimentos motores, daí a importância da investigação do enfermeiro durante a elaboração dos DE.

Um dos problemas de saúde enfrentados pela população idosa é a ocorrência de quedas. Dentre as condições relacionadas a este evento, destaca-se a mobilidade física prejudicada. Salienta-se ainda que a ocorrência de agravos neurológicos como o AVE, vinculada a outros fatores, potencializam o risco de quedas (Costa, Oliveira, Moreira, Cavalcante, \& Araujo, 2010).

As quedas geralmente ocorrem devido a um conjunto de fatores, podendo ser intrínsecos (desequilíbrio, fraqueza, doenças crônicas entre outros) e extrínsecos (local com baixa iluminação, presença de tapetes escorregadios). Ainda, diversos autores apontam o AVE como um dos fatores de risco mais relevantes para o risco de quedas, tendo em vista que afeta o equilíbrio e marcha, potencializando dessa forma os fatores extrínsecos (Costa, 2017).

Partindo do pressuposto que as pessoas mais velhas são as mais acometidas pelo AVE e que a ocorrência de quedas é multifatorial em idosos, um episódio de AVE influencia no aumento dos fatores de risco para quedas, ainda, as pessoas acometidas pelo AVE apresentam quedas com mais frequência do que outras pessoas que não tiveram a doença e possuem a mesma idade. Demonstrando assim que as quedas estão altamente relacionadas a mobilidade física prejudicada decorrente do AVE (Costa, 2017).

Ao verificar quais foram as características definidoras mais presentes dentro do diagnóstico de MFP, encontrou-se a redução nas habilidades motoras finas e grossas, dificuldade para virar-se, alteração na marcha e movimentos descoordenados. Sendo a redução nas habilidades motoras finas e grossas também uma das características mais presentes no estudo de Moreira et al. (2016), onde avaliou o PE de 38 pacientes em tratamento ambulatorial em um Hospital de referência no nordeste brasileiro.

Estes dados vão ao encontro com estudo realizado por Lescano, e Santos (2016), com paciente portador de sequela de AVE que frequentava a Clínica de Fisioterapia do Centro Universitário da Grande Dourados, onde foi realizado levantamento de diagnósticos de enfermagem para este paciente. O diagnóstico de MFP foi um dos elencados e teve como características definidoras a amplitude limitada de movimento, alterações na marcha e movimentos lentos.

Conforme o estudo realizado por Lescano, e Santos (2016), com paciente portador de sequela de AVE em uma Clínica de Fisioterapia do Centro Universitário da Grande Dourados, o qual realizou o levantamento de diagnósticos de enfermagem, direcionados a este paciente, o diagnóstico de MFP foi um dos elencados e teve como característica definidora a amplitude limitada de movimento, mudanças na marcha e movimentos lentos.

Os fatores relacionados força muscular diminuída e alteração na função cognitiva foram os mais citados dentro do diagnóstico de MFP, dados semelhantes com os encontrados por Costa, Oliveira, Moreira, Cavalcante, e Araújo (2010), onde 121 pacientes foram avaliados no período em que encontravam-se em processo de reabilitação pós AVE, no qual o fator relacionado "força muscular diminuída" foi o mais presente na população com diagnóstico de MFP. Já no estudo de Lescano, e Santos (2016), o fator relacionado prejuízos neuromusculares, foi o mais citado.

Diante dos dados encontrados, observa-se que o enfermeiro precisa trabalhar de forma que proporcione uma assistência direcionada as necessidades dos pacientes com AVE, com práticas voltadas a orientar e ensinar, considerando as limitações de cada paciente decorrentes da doença, bem como as prováveis mudanças nos hábitos de vida. Ele deve também deve estar atento ao levantamento de diagnósticos de enfermagem precisos, relativos as reais necessidade manifestadas pelo 
paciente, com o propósito de tornar viável a realização das ações de prevenção, manutenção e reabilitação da saúde e qualidade de vida desse paciente (Costa, Oliveira, Moreira, Cavalcante, \& Araújo, 2010).

Cita-se como principais limitações deste estudo que durante o período de pandemia da Covid-19 (a partir de março de 2020), a rotina na unidade acabou sendo afetada, pois ocorreu um aumento na demanda de atendimentos e internações, ainda teve o fechamento de um dos setores do hospital, devido à necessidade de profissionais para atuarem nos setores de internação de pacientes acometidos pelo Covid-19 (enfermaria e UTI).

Com isso, os pacientes que antes estariam no setor que ocorreu o fechamento, tiveram que ser realocados para outros setores do hospital, incluindo a neurologia devido à falta de leitos, causando dessa forma a ocupação de leitos de pacientes neurológicos por pacientes cirúrgicos/ortopédicos, assim como devido a unidade lotada, muitos pacientes neurológicos acabaram sendo internados em outros setores, sendo estes perdidos da amostra.

\section{Conclusão}

Com base no estudo, evidenciou-se que o sexo masculino foi o mais acometido e a média de idade dos pacientes foi de 64 anos, com alta incidência da hipertensão e a maioria apresentou AVE isquêmico.

Dos prontuários analisados, 50\% apresentavam o processo de enfermagem realizado, e destes, o diagnóstico de enfermagem mobilidade física prejudicada constava em 17,5\%. As principais características definidoras relacionadas ao diagnóstico de enfermagem mobilidade física prejudicada foram redução nas habilidades motoras finas e grossas, dificuldade para virar-se, alteração na marcha e movimentos descoordenados. Os fatores relacionados força muscular diminuída e alteração na função cognitiva foram os mais citados.

Ressalta-se a importância da enfermagem na avaliação clínica dos pacientes, estarem atentos as limitações apresentadas para o estabelecimento de diagnósticos de enfermagem precisos. Para isso necessita-se mais estudos para investigação da mobilidade prejudicada em pessoas com AVE, assim como pesquisas voltadas à escalas para avaliação da mobilidade física, afim de melhorar a qualidade de vida de pacientes com AVE e acelerar o processo de reabilitação.

Instiga-se por fim o desenvolvimento de mais pesquisas voltadas a este público, através de pesquisa em campo, realizando anamnese e exame físico, permitindo assim um olhar mais detalhado referente aos sinais e sintomas apresentados, o tempo entre o início dos sintomas e a busca por atendimento. Bem como a realização ou não de tratamento trombolítico e sua eficácia quanto à recuperação dos déficits apresentados, ainda sendo possível realizar uma comparação entre aqueles que realizaram o tratamento e os que não foi possível.

\section{Referências}

Albuquerque, E. S., Florêncio, R. S., Moreira, T. M. M., Lima, S. C. S., Mattos, S. M., \& Ribeiro, D. C (2020). Fatores de risco para acidente vascular encefálico em jovens: Uma comparação entre homens e mulheres. Pesquisa, Sociedade e Desenvolvimento, 9 (8), e824986503. https://doi.org/10.33448/rsdv9i8.6503

Araujo, L. P. G., Souza, G. S., Dias, P. L. R., Nepomuceno, R. M., \& Cola, C. S. D (2017). Principais fatores de risco para o acidente vascular encefálico e suas consequências: uma revisão de literatura. Revista Interdisciplinar Pensamento Científico, 3(1), 283-296. http://www.reinpec.org/reinpec/index.php/reinpec/article/view/155

Barella, R. P., Duran, V. A. A., Pires, A. J., \& Duarte, R. O (2019). Perfil do atendimento de pacientes com acidente vascular cerebral em um hospital filantrópico do sul de Santa Catarina e estudo de viabilidade para implantação da unidade de AVC. Arquivos Catarinenses de Medicina, 48(1), 131-143. https://docs.bvsalud.org/biblioref/2019/10/1023423/432-1341-2-rv.pdf

Cavalcante, T. F., Nemer, A. P. L., Moreira, R., \& Ferreira, J (2018). Intervenções de enfermagem ao paciente com acidente cerebrovascular em reabilitação. Revista de Enfermagem UFPE on line, 12(5), 1430-1436. https://doi.org/10.5205/1981-8963-v12i5a230533p1430-1436-2018

Costa, A. G. S., Kumakura, A. R. S. O., Araujo, T. L., Castro, N. B., Silva, V. M., \& Lopes, M. V. O (2017). Stroke and risk factors for falls in elderly individuals. Revista da Rede de Enfermagem do Nordeste, 18(5), 663-670. https://doi.org/10.15253/2175-6783.2017000500014 
Costa, A. G. S., Oliveira, A. R. S., Moreira, R. P., Cavalcante, T. F., \& Araujo, T. L (2010). Identificação do risco de quedas em idosos após acidente vascular encefálico. Escola Anna Nery, 14(4), 684-689. http://dx.doi.org/10.1590/S1414-81452010000400005

Creswell, J. W (2010). Projeto de pesquisa: método qualitativo, quantitativo e misto: Artmed Editora.

Gaspari, A. P., Cruz, E. D. A., Batista, J., Alpendre, F. T., Zétola, V., \& Lange, M. C (2019). Preditores de internação prolongada em Unidade de Acidente Vascular Cerebral (AVC). Revista Latino-Americana de Enfermagem, 27: e3197. http://dx.doi.org/10.1590/1518-8345.3118.3197

Gil, A. C (2019). Como Elaborar Projetos de Pesquisa: Atlas.

Gonçalves, J. L., Feitosa, E. S., \& Borges, R. T (2019). Perfil epidemiológico de vítimas de acidente vascular encefálico em um hospital de referência do Ceará/Brasil. Revista Interdisciplinar, 12(2), 92-103. https://revistainterdisciplinar.uninovafapi.edu.br/index.php/revinter/article/view/1219/pdf_426

Herdtman, T. H., \& Kamitsuru, S (2018). Diagnósticos de Enfermagem da NANDA-I - Definições e Classificações: Artmed Editora.

Knechtel, M. R (2014). Metodologia da pesquisa em educação: uma abordagem teórico-prática dialogada: Intersaberes.

Lescano, G., \& Santos, J. R (2016). Relato de caso: Principais diagnósticos de enfermagem para o portador de sequela de acidente vascular encefálico. Enfermagem Brasil, 15(3), 152-159. https://portalatlanticaeditora.com.br/index.php/enfermagembrasil/article/viewFile/182/1540

Lima, A. C. M. A. C. C., Silva, A. L., Guerra, D. R., Barbosa, I. V., Bezerra, K. C., \& Oriá, M. O. B (2016). Nursing diagnoses in patients with cerebral vascular accident: an integrative review. Revista Brasileira Enfermagem, 69(4), 738-45. http://dx.doi.org/10.1590/0034-7167.2016690423i

Lima, A. L. (2020). Seis sequelas mais comuns do AVC. Obtido em https://www.tuasaude.com/sequelas-de-avc/

Lunardi, A. C. (2020). Manual de Pesquisa Clínica aplicada à Saúde: Blucher.

Ministério da Saúde (BR). Acidente vascular cerebral. 2020. https://www.gov.br/saude/pt-br/assuntos/saude-de-a-a-z-1/a/avc-o-que-e-causas-sintomastratamentos-diagnostico-e-prevencao

Miranda, M. (2020). AVC Hemorrágico. http://www.ineuro.com.br/para-os-pacientes/avc-hemorragico/

Moreira, R. P., Araujo, T. L., Lopes, M. V. O., Cavalcante, T. F., Guedes, N. G., Chaves, E. S., Portela, R. C., \& Holanda, R. E (2016). Validação clínica do resultado de enfermagem mobilidade em pacientes com acidente vascular cerebral. Revista Gaúcha de Enfermagem, 37(4):e54688. http://doi.org/10.1590/1983-1447.2016.04.54688

Mourão, A., Vicente, L. C. C, Chaves, T. S., Sant`Anna, R. V., Meira, F. C., Xavier, R. M. B., Tanure, M., Souza, L., \& Teixeira, A (2017). Perfil dos pacientes com diagnóstico de AVC atendidos em um Hospital de Minas Gerais credenciado na linha de cuidados. Revista Brasileira de Neurologia, 53(4), 1216. https://revistas.ufrj.br/index.php/rbn/article/view/14634

Musse, N. (2018). AVC Isquêmico e Hemorrágico! Qual a diferença? https://www.drneifmusse.com.br/avc-isquemico-e-hemorragico-qual-adiferenca/\#: :text=O\%20AVC\%20Hemorr\%C3\%A1gico\%20\%C3\%A9\%20conhecido,\%C3\%A0\%20morte\%2C\%20nos\%20mais\%20graves

Nunes, D. L., De S., Fontes, W. Dos S., \& Lima, M. A. De. (2016). Cuidado de enfermagem ao paciente vítima de acidente vascular encefálico. Revista Brasileira de Ciências da Saúde, 21(1), 87-96. https://doi.org/10.22478/ufpb.2317-6032.2017v21n1.24003

Oliveira, M. M. (2018). Como Fazer Pesquisa Qualitativa: Vozes.

Oliveira, B. L. C. A., Cardoso, L. F. C., Dominice, R. O., Corrêa, A. A. P., Fonseca, A. E. C., Moreira, J. P. L., \& Luiz, R. R (2020). A influência da Estratégia Saúde da Família no uso de serviços de saúde por adultos hipertensos no Brasil. Revista Brasileira de Epidemiologia, 23 , e200006. http://dx.doi.org/10.1590/1980-549720200006

Pereira, A. S., Shitsuka, D. M., Parreira, F. J., \& Shitsuka, R (2018). Metodologia da pesquisa científica. UFSM. https://repositorio.ufsm.br/bitstream/hand le/1/15824/Lic_Computacao_Metodologia-Pesquisa-Cientifica.pdf?sequence=1

Pereira, M. M. M., Cézar, E. S. R., Pereira, V. C. L. S., Braga, L. S., Espínola, L. L., \& Azevedo, E. B (2015). Saúde do homem na atenção básica: análise acerca do perfil e agravos à saúde. Revista de Enfermagem UFPE on line, 9(1), 440-447. http://dx.doi.org/10.5205/reuol.5221-43270-1-RV.0901 supl201524

Rosin, J., Matos, F. G. O. A., Alves, D. C. I., Carvalho, A. R. S., \& Lahm, J. V (2016). Identificação de diagnósticos e intervenções de enfermagem para pacientes neurológicos internados em hospital de ensino. Ciência, Cuidado e Saúde, 15(4), 607-615. https://doi.org/10.4025/cienccuidsaude.v15i4.31167

Santos, L. B., \& Waters, C (2020). Perfil epidemiológico dos pacientes acometidos por acidente vascular cerebral: revisão integrativa. Brazilian Journal Of Development, 6(1), 2749-2775. http://dx.doi.org/10.34117/bjdv6n1-198

Silva, R. A. P. (2020). Seis doenças que mais afetam a saúde do homem. https://www.clinicaceu.com.br/blog/doencas-que-mais-afetam-a-saude-do-homem/

Teixeira, D. B. S., \& Cruz, S. P. L (2016). Atenção à saúde do homem: análise da sua resistência na procura dos serviços de saúde. Revista Cubana de Enfermería, 32(4), 126-136. http://www.revenfermeria.sld.cu/index.php/enf/article/view/985/209 